\title{
Improved Fractographic Strength Estimates Based on Surface Profilometry
}

\author{
Lingyue $\mathrm{Ma}^{1}$ and Roberto Dugnani ${ }^{1}$ \\ ${ }^{1}$ University of Michigan, Shanghai Jiao Tong University Joint Institute, UM-SJTU JI, 800 Dongchuan Road, Shanghai, 200240, China
}

\begin{abstract}
Fractography is a valuable method that uses post-mortem topographical information to estimate the stress field near the fracture origin and help establish the root cause of failures. Typically, in glass and ceramics the mirror radius is one of the features sought for by fractographers since its length could be empirically related to the sample's strength. The mirror radius is usually subjectively estimated by fractographers though microscopy measurements. Nonetheless, variations in the estimates introduced by inconsistent viewing modes and the subjectivity of observers could lead to substantial errors even when standard protocols such as ASTM C1678 were followed. In this manuscript, a novel method combining a fracture mechanics model describing the mist formation in silicate glasses with profilometry data carried out by confocal laser scanning microscope is introduced. The new method was shown to be able to objectively establish the mirror-mist boundary. Furthermore, it was found that the proposed technique was repeatable within $2 \%$ regardless of the magnification or imaging mode used. Whereas the average strength estimated per ASTM C1678 by eight individual observers was influenced by both the magnification and the imaging mode used and displayed standard deviation of over $3 \%$.
\end{abstract}

\section{Introduction}

Fractography is often used to estimate the fracture strength of brittle materials by analyzing revealing fracture patterns. In silicate glass and ceramics, these telltale surface marks include a relatively smooth region surrounding the fracture origin, named the "mirror region", a coarse, hazy "mist region" adjacent to mirror region, and a region with feather-like, non-coplanar segments called the "hackle region".

For over half a century, fractographers have been estimating the strength of the fractured samples by measuring the distance from the fracture origin to the boundary of the mirror/mist region. This characteristic length scale, known as the mirror radius or inner-mirror radius, could correlated to the strength of the material using Orr's equation [1]

$$
\sigma_{f}=\frac{A}{\sqrt{R}}+C \text {. }
$$

Where $R$ is the mirror radius, $A$ and $C$ are linearly regressed constants, and $\sigma_{f}$ is the fracture strength. The constant, $A$ is often referred to as the mirror constant. Orr's original work did not include $C$, but its use has become standard especially for $C>10 \mathrm{MPa}$, as recommended by both NIST and ASTM standards [2, 3].

The process of measuring mirror radius conventionally is subjective and hence it naturally leads to estimating error. In order to alleviate this problem both ASTM standard [2] and the NIST guide [3] introduced standard procedures for mirror radius measurements.
Nonetheless, even when carefully following the directives outlined in the standard, the measuring process inevitably leads to variations due to the subjective nature of the process. Additional variations in the estimations are caused by factors such as the type of viewing method, the illumination used, and the magnification used [4].

Arguably, the major source of errors when investigating fractures is introduced by the lack of a clear definition of what constitutes the mirror/mist boundary [5]. Normally observers optically discern the mist as the relative rough surface where - based on their personal perception - the visible light begins to be scattered by the increasingly rougher surface. This suggests that the threshold of the roughness should be a fraction of the range given by the wavelength of visible light (i.e., $0.39-0.80 \mu \mathrm{m}$ ) [4], yet no clear demarcation actually exists and no clear arbitrary roughness threshold has been set by neither NIST nor ASTM standards. Consequently, it is expected that the mirror radius be affected by viewing methods and in general it was found that researchers using non-optical tools suggested lower roughness values to describe the mirror-mist boundary [6-9].

In this manuscript, a novel method is described to obtain accurate and repeatable estimations of the mirror radius. The method combines knowledge of the theoretical behaviour describing the mist formation in silicate glasses based on fracture mechanics principles with profilometry data obtained by confocal laser scanning microscope (CLSM). The novelty of the method 
consists in combining advanced visualization technique with the most current understanding of the brittle fracture behaviour.

\section{Methods}

In order to consistently establish the length of the mirror radius, a method combining the theoretical description of the mirror-mist boundary locus with profilometry data of the fracture surface was used. In this section, the numerical algorithm used to manipulate the fracture surface profilometry data was described in detail.

The profilometry scan of the fracture surfaces were carried out by CLSM (Zeiss LSM 700/Olympus PMG 3) with the operating parameters summarized in table 1 . The methods to determine the two extremities defining the mirror radius, i.e. the origin, and the mirror-mist boundary were described separately.

Table 1. Operating parameters for CLSM.

\begin{tabular}{ccccc}
\hline $\begin{array}{c}\text { Laser } \\
\text { wavelength }\end{array}$ & $\begin{array}{c}\text { Laser } \\
\text { Intensity }\end{array}$ & Pinhole & $\begin{array}{c}\text { Channel } \\
\text { Gain }\end{array}$ & $\begin{array}{c}\text { Digital } \\
\text { offset }\end{array}$ \\
\hline $405 \mathrm{~nm}$ & 1.2 & $1 \mathrm{AU}^{1}$ & $\sim 350$ & 1.0 \\
\hline
\end{tabular}

\subsection{Samples preparation}

Two sets of glass plates were tested by four-points bending tests (4PTB) according to ASTM standard C158-02 [10]. The first set included alumino-silicate glass of $0.7 \mathrm{~mm}$ thick, and the second set included soda-lime glass of $2 \mathrm{~mm}$ thick. The tests were conducted on a MTS testing system (model 45) with a $50 \mathrm{kN}$ load cell (resolution of $0.01 \mathrm{~N}$ ), outer/inner span of $200 / 100 \mathrm{~mm}$ and rollers' diameter of $10 \mathrm{~mm}$. The fractured surfaces were first scanned by CLSM and then imaged by conventional optical microscopy both in bright-field and differential interference contrast (DIC).

\subsection{Origin determination}

According to ASTM standard [2], the mirror radius on fractures with origins away from the edges could be obtained as half the length between the two mirror-mist boundaries at the stress-free surface. Nonetheless, for samples with corner crack origins or near-the-edge origins, the length of the mirror radius is not as straightforward to obtain as it requires knowledge of the location of the fracture origin.

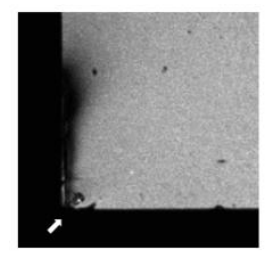

(a)

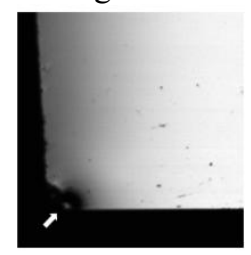

(b)

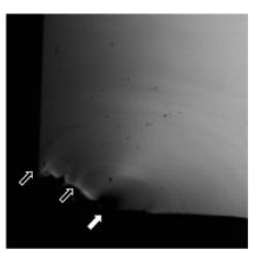

(c)
Figure 1. Origins under CLSM from different glass samples fractured by 4PTB.

\footnotetext{
11.01 Airy Units $=13.0 \mu \mathrm{m}$ section.
}

Fig. 1 shows various corner fracture origins from samples fractured by 4 PTB with white arrows pointing at the origin. When the fracture origins were not very obvious when inspected by bright field, different illumination method such as DIC, or non-optical methods such as SEM, could help discern the origin. Often, during slow crack growth the initial flaw might form an elliptic or semi-circular shadowed ring around the origin which will help locate its position (Fig. 1a). The presence of a flaw on a plane other than the fracture plane could also be distinguishable by CLSM as a shady region (Fig. 1b). Other surface markings like hackles and Wallner lines (hollow arrows in Fig. 1c) could also help establish the location of origin but might require some understanding of the dynamic behavior of the crack.

The exact placement of the fracture origin in general was expected to remain to some degrees of arbitrary. Nonetheless, the use of appropriate techniques could help reduce the uncertainty about the fracture origin location. Further discussion on how to determine the fracture origins could be found in the NIST guidelines [3].

\subsection{Mirror-mist boundary determination}

In this section, a novel method to establish the mirror radius based on CLSM profile images of the fracture surface is introduced. It should be noted, that the use of profilometry data to estimate fractographical features is not new. For instance, Duckworth et al. [6] used a Talysurf surface profilometer to trace the fracture surface along a direction parallel to the tension surface. The trace was then used to define the mirror boundary based on relative surface roughness. More recently, López-Cepero et al. [11] applied CLSM to study fracture profiles of erbia-stabilized zirconia and ruby fibers. Nonetheless, both these works established the length of the mirror region based on a threshold near the free surface rather than using a larger portion of information available from the fracture surface.

As the transition from mirror to mist region is a gradual progress caused by the gradual increase in surface roughness, an arbitrary roughness threshold had to be set to uniquely establish an arbitrary yet reputably measurable "mirror-mist boundary". In this work, the onset of the mist was chosen in the range $0.4-0.8 \mu \mathrm{m}$ (i.e. the wavelength of visible light) based on the arguments similar to Quinn [4].

The surface contour at the stress-free surface is shown in Fig. 2, and basic fractographic feature could be inferred by the degree of roughness of the surface contour.

Detecting the mirror-mist boundary region from a fracture profile could be facilitated if the expected shape of the mirror-mist boundary could be described analytically. In particular, an analytical description of the mirror-mist boundary could enable the determination of the mirror radius using larger portions of the fracture surface rather than simply relying on limited information at the free-edge (which might also be affected by residual stresses). In this work the geometrical description of the mirror-mist boundary was based on fracture mechanics 
(a)

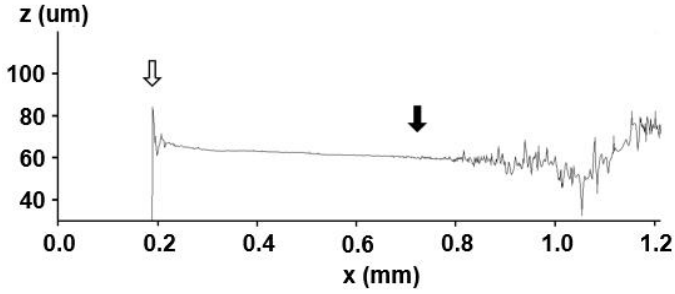

(b)

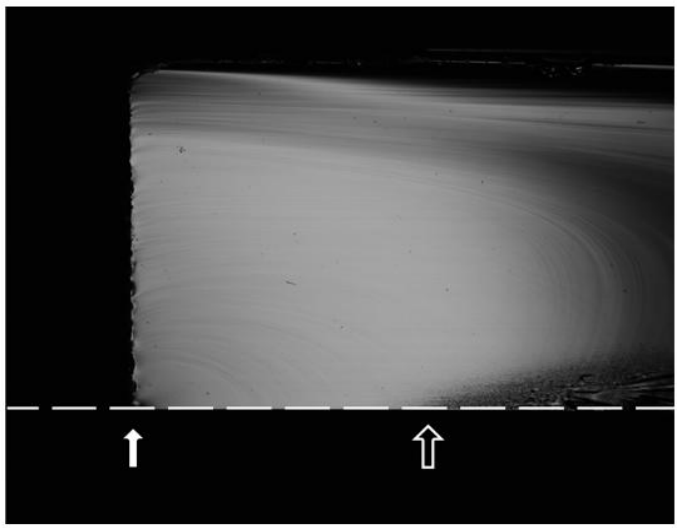

Figure 2. Profilometry contour (a) taken from the sample on the free edge (b, white dash line). White arrows denote the origin, black arrows denote the onset of mist.

analysis conducted by Dugnani and Zednik [12]. Dugnani and Zednik indicated that the geometric shape of the mirror-mist boundary for a brittle sample fractured in either tension or bending could be described by the equation of a conic section. In particular, the mirror-mist boundary shape near the free surface for samples fractured in bending was expected to follow an elliptical curve for values of $R / H<0.2$, a parabolic curve for $0.2<R / H<0.4$, and a hyperbolic curve for values of $R / H>0.4$. The shape of the mirror-mist boundary region for the soda-lime glass samples fractured in this work appeared to follow the trends described by the work of Dugnani and Zednik [12]. Fig. 3 (b) though (f) show examples of fracture surfaces for values of $R / H$ corresponding to the elliptic, the parabolic, and the hyperbolic range. Also Fig. 3(a) shows a contour of the corresponding expected mirror-mist boundary shape for various values of $R / H$ based on the theoretical work of Dugnai and Zednik [12]. With these premises, an efficient computational algorithm to obtain the mirror-mist boundary could be developed.

Fig. 4 shows the flowchart describing the methodology to assess the mirror radius. First, the surface roughness data in an area including the origin and the mirror-mist boundary were obtained by CLSM. Next both the estimated mirror-mist boundary radius, $R_{e}$ (e.g., white dash line in Fig. 5b), and the slope of the mirror-mist boundary at the free surface, $\theta_{m}$, were estimated visually. Alternatively, for $R_{e}>0.16 \mathrm{H}$, the approximate slope of the mirror-mist angle, $\theta_{m}$, could be approximated using the relationship suggested by Dugnani and Zednik [13]:

$$
\frac{H}{R_{e}}=2 \tan \left(1.1 \theta_{m}\right)+0.25
$$

After estimating the mirror radius, a rectangular region of height $H / 10$ and length $2 / 5 R_{e}$ was set at a distance of $0.05 \mathrm{H}$ from the origin and the free surface (a)
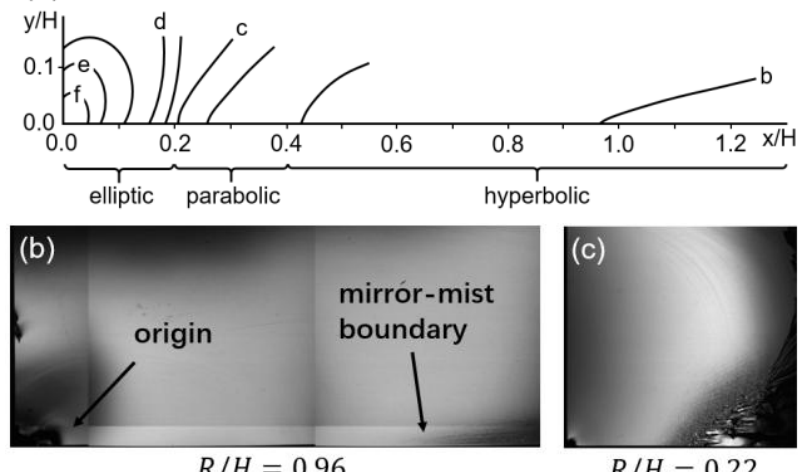

$R / H=0.22$

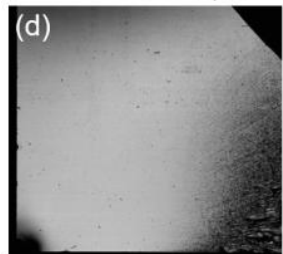

$R / H=0.164$

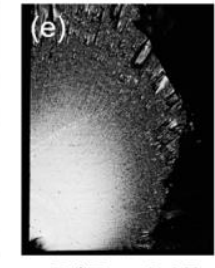

$R / H=0.07$

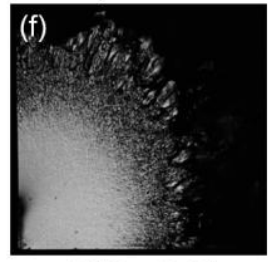

$R / H=0.05$
Figure 3. Mirror-mist boundaries under different fracture strength by 4 PTB.

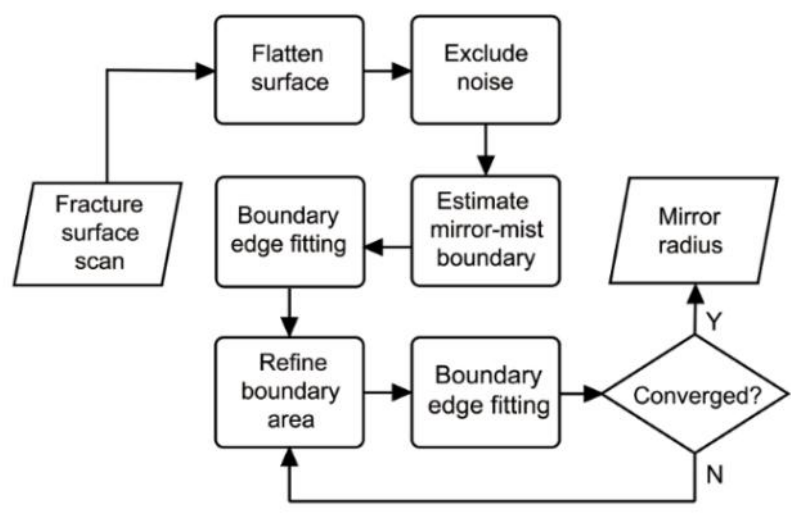

Figure 4. Flowchart for the algorithm used to determine the mirror radius.

was selected to flatten the fracture surface and establish a reference plane (Fig. 5a). Next, all portions of the image that displayed a height difference greater than $30 \mu \mathrm{m}$ from the reference plane were excluded. A parallelogram (black in Fig. 5b) with one edge centred at $R_{e}$, and height $0.1 \mathrm{H}$, width $0.5 R_{e}$, and slanted edges at an angle $\theta_{m}$ from the free surface was then defined. The root-mean-square (RMS) of the surface height was calculated within the parallelogram and all the locations corresponding to surface roughness in range of $0.4-0.8 \mu \mathrm{m}$ were selected (white dots in Fig. 5c or black in Fig. 5d) and the remaining area discarded. A conic curve (black, Fig. 5c) was regressed through the points corresponding to the surface roughness in the range of interest. The type of conic section equation used to regress the data was determined based on the value of $R_{e} / H$, as explained in Dugnani and Zednik [12]. The curve obtained from the initial data regression (white, Fig. 5e) was used to define a new parallelogram with two curved edges and centred at the intersection between the fitted conic equation and the free surface (black, Fig. 5e). Subsequently, a new conic curve was generated, a new value of $R_{i}$ obtained 
and a new parallelogram defined. These steps were repeated until convergence which was achieved when the absolute value of the average difference between two subsequent iterations of the fitted conic curves was less than $1 \%$ (converged white curve in Fig. 5f).
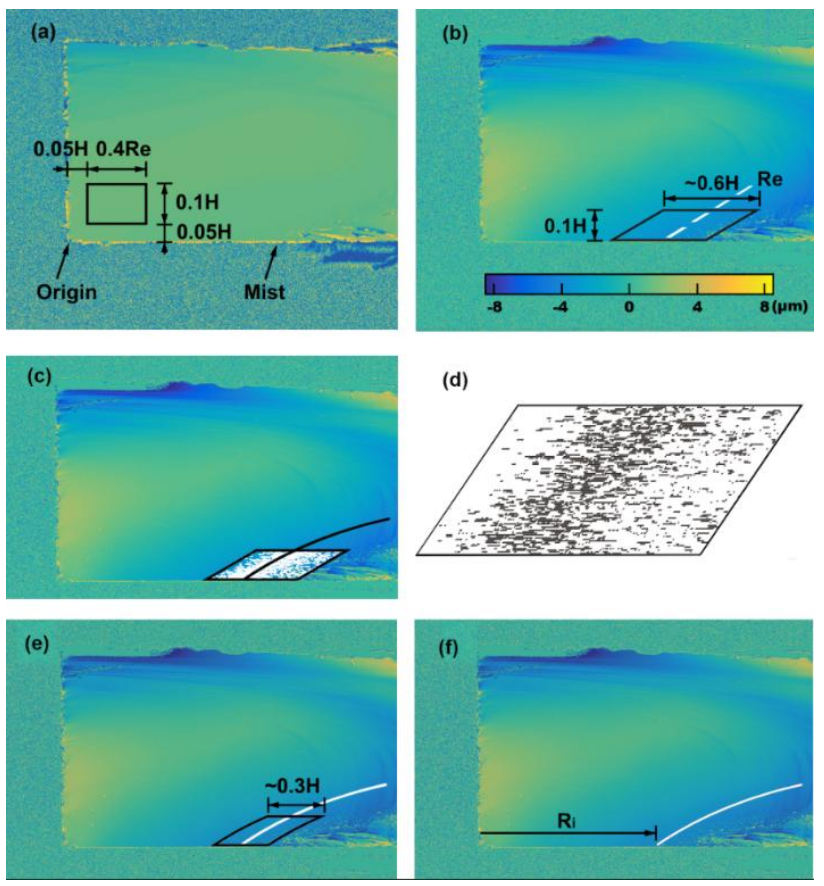

Figure 5. Main steps for algorithmic method.

\section{Results}

The mirror-mist boundaries were established using the profilometry-based measurements on twelve specimens using bright-field illumination and on six specimens using DIC. The constants $A$ and $C$ in Eq. (1) were obtained separately for both the proposed method and the traditional microscopic method using bright-field illumination measurements as suggested by the ASTM standard [2]. Additionally, mirror radii from the same specimens were also estimated by eight individual observers using standard optical microscopic technique and the profilometry-based method was repeated around eight times for each sample. These results were used to compare the repeatability of the measurements.

A relative stress estimation errors (RSE) were defined as:

$$
\text { RSE }=\frac{\left(\sigma_{\text {estimated }}-\sigma_{\text {applied }}\right)}{\sigma_{\text {applied }}} \cdot 100 \% .
$$

The value of RSE was computed for both the newly developed method and for the standard optical method of defining the mirror radii. In Eq. (3), $\sigma_{\text {applied }}$ stood for the recorded fracture strength from $4 \mathrm{PTB}$, and $\sigma_{\text {estimated }}$ for the strength estimate by the measured mirror radius using Eq. (1). RSE represented the relative estimating error of the strength.

Table 2 shows different RSE from the profilometry scans and for the same samples based on optical images taken under the same viewing conditions. For the same set of samples, the deviations of RSE by optical microscope were larger than $3 \%$, while for the profilometry-based algorithm deviations were less than $2 \%$. Furthermore, DIC displayed a difference in mean RSE value up to $1.75 \%$ for optical method but it was nearly unchanged for the profilometry-based method.

Table 2. Selected RSE results for profilometry-based method vs. RSE from optical microscopy by eight observers.

\begin{tabular}{cccc}
\hline $\begin{array}{c}\text { Viewing } \\
\text { modes }\end{array}$ & $\begin{array}{c}\text { Samples } \\
\text { tested }\end{array}$ & $\begin{array}{c}\text { RSE } \\
\text { (optical } \\
\text { microscopy) }\end{array}$ & $\begin{array}{c}\text { RSE } \\
\text { (profilometry- } \\
\text { based method) }\end{array}$ \\
\hline $\begin{array}{c}\text { Bright-field } \\
10 \times\end{array}$ & 12 & $0.00 \% \pm 3.14 \%$ & $0.00 \% \pm 1.84 \%$ \\
DIC $10 \times$ & 4 & $1.75 \% \pm 3.51 \%$ & $0.11 \% \pm 1.78 \%$ \\
DIC $20 \times$ & 2 & $0.72 \% \pm 3.16 \%$ & $0.05 \% \pm 1.86 \%$ \\
\hline
\end{tabular}

\section{Discussion}

Profilometry measurements carried out on twelve samples were compared with the corresponding optical methods for two viewing methods (i.e., bright field and DIC) and two magnification factors (i.e., 10x and 20x). Eight individual observers estimated the mirror radius per ASTM C1678 by optical microscopy (table 2).

Based on the analysis performed, the profilometry-based method was more repeatable than the optical methods.

In optical methods, observers gave different results due to their own interpretation of the location of the mirror-mist boundary, and the standard deviations in the reported strength for the data set considered were more than $3 \%$. The reported results were also affected by the viewing modes and magnifications. For the profilometry-based method, the RSE deviations were within $2 \%$. Furthermore, the mean value of RSE was higher for the DIC compared to bright-filed imaging, whereas it was nearly unchanged for the profilometry-based method.

No low-strength samples were examined in this manuscript, but the merit of this method could be more obvious in analyzing low-strength samples, since relative errors introduced by common viewing modes would be magnified on the low-strength basis. The mist could be faint near the free surface for low-strength samples, thus would lead to a large uncertainty in methods based on optical estimations. Nonetheless, the fitting process in the profilometry-based method could amend the boundary based on roughness and the clearer part of the boundary along with the curve shape indicated by Dugnani and Zednik.

The provided algorithmic method could have a small deviation (less than 2\%) when defining the mirror-mist boundary, but the difficulty existed in dealing with very thin and flat mist, since the less points of the area of interest analysed, the more uncertainty existed in the final fitting result. Subjectivity also remained in the determination of mirror radius due to the complexity in defining the origin. The profilometry-based method in this manuscript introduced relatively stable and repeatable way to determine the mirror radius and to 
estimate the strength compared to conventional microscopy method.

\section{Future Work}

In this manuscript, an algorithmic method for mirror radius determination was provided based on the profilometry data collected by CLSM. Although the method was capable of identifying the mirror-mist boundary, and measuring the mirror radius in a more repeatable way compared to optical estimations, future work is recommended. In particular, future work should include:

(1) The fracture origin was determined with some degree of subjectivity. Further work should be carried out to estimate the origin more objectively based on the surface marking on the fracture surface (e.g., Wallner lines).

(2) One of the premises of this method was the shape of the mirror-mist boundaries followed a conic relation. This work conducted by Dugnani and Zednik [12] was based on the straight crack solution for the crack propagation. A more appropriate dynamic model should be implemented to better explain the loci of the mirror-mist boundaries.

\section{Conclusion}

In this manuscript, an algorithmic method was introduced to determine the mirror-mist boundary based on profilometry data obtained from the confocal laser scanning microscope. Fracture surface scans were obtained by confocal laser scanning microscope for the analysis of surface height and roughness. The mirror-mist boundary was determined based on the threshold of the roughness transition on the fracture surface. Comparison was made on twelve samples applying both profilometry-based method and optical microscopy conducted by eight individual observers per the ASTM C1678. Compared to the optical microscopy methods, the profilometry-based method displayed approximately $2 \%$ repeatability for the inner mirror measurements whereas conventional methods showed more than 3\%. More importantly, the estimate from profilometry-based method was not affected by either viewing mode or magnification unlike the optical microscopy method. The profilometry-based analysis has then been shown to significantly reduce errors commonly introduced by the subjective interpretation of the mirror-mist boundary position, viewing modes, and magnification factors.

\section{Acknowledgement}

The authors would like to extend their gratitude to Ying Wang, Xiangxing Lv, Zhengdong Shan, Weizi Li, Huan Sun from UM-SJTU JI, and Anthony Moulins from ETS in Montréal for helping collecting data. They also thank Anthony Moulins for generating some of the profilometry data.

\section{References}

1. L. Orr. Mater. Res. Stand. 12 (1972)

2. ASTM C1678-10, Standard Practice for Fractographic Analysis of Fracture Mirror Sizes in Ceramics and Glasses (2015)

3. G.D. Quinn. Fractography of Glasses and Ceramics V: Ceramic Transactions. 199 163-187 (2007)

4. G.D. Quinn: NIST Recommended Practice Guide: Fractography of Ceramics and Glasses. NIST SP 960-16 (2016)

5. J. Mecholsky, S. Freiman: Determination of fracture mechanics parameters through fractographic analysis of ceramics, in Fracture Mechanics Applied to Brittle Materials: ASTM International, 136-150 (1979)

6. W. Duckworth, D. Shetty, A. Rosenfield. Glass Technol. 24 263-273 (1983)

7. D. Hull. J. Mater. Sci. 31 1829-1841 (1996)

8. D. Hull: Fractography: observing, measuring and interpreting fracture surface topography. Cambridge University Press (1999)

9. D. Kulawansa, L. Jensen, S. Langford, J. Dickinson, Y. Watanabe. J. Mater. Res. 9 476-485 (1994)

10. ASTM C158-02, Standard Test Methods for Strength of Glass by Flexure (Determination of Modulus of Rupture) (2012)

11. J. Lopez-Cepero, A. De Arellano-Lopez, J. Quispe-Cancapa, J. Martínez-Fernández. 19 13-15 (2005)

12. R. Dugnani, R.J. Zednik. Eng. Fract. Mech. 165 87-97 (2016)

13. R. Dugnani, R.J. Zednik. 97 3853-3856 (2014) 